\title{
Fatores que propiciam a gravidez na adolescência em uma unidade de referência especializada materno infantil na região Norte do Brasil: um estudo piloto
}

Factors that provide pregnancy in adolescence in a specialized reference unit for children's maternal in the North region of Brazil: a pilot study

Factores que sondean el embarazo en adolescencia en una unidad de referência especializada para maternos infantiles en el Norte de Brasil: un estudio piloto

Ananda Maciel Paiva ${ }^{1 *}$, Ana Laura da Costa Medeiros ${ }^{1}$, Ataide José Mendes Netto², Brunno Rôlo, Cenilde da Costa Araújo ${ }^{1}$, Danielli Valeria Diniz Felisberto ${ }^{1}$, Natalia Tavares Carvalho ${ }^{1}$, Rayssa Raquel Araújo Barbosa ${ }^{3}$, Sheila Mara Dias ${ }^{4}$, Tamara Catarino Fernandes.

\section{RESUMO}

Objetivo: Analisar os fatores de risco para a gravidez em adolescentes na faixa etária de 10 a 19 anos na Unidade de Referência Especializada Materno Infantil (UREMIA); verificar a utilização de métodos contraceptivos na população acima especificada; verificar o perfil socioeconômico da família das adolescentes. Métodos: Estudo quantitativo descritivo observacional, que visa o levantamento de dados sobre os fatores que levam às adolescentes a engravidarem. A população desse estudo é composta por adolescentes grávidas, com idade entre 10 e 19 anos. Resultados: 30,4\% tiveram a sexarca aos 13 e 14 anos, 39,3\% responderam que são solteiras. A maioria das adolescentes tem conhecimento sobre IST's $(72,7 \%)$ e $54,1 \%$ destas orientações foram passadas principalmente na escola. $54,6 \%$ afirmaram fazer uso de método contraceptivo. $48,8 \%$ possuem ensino fundamental incompleto. $45,6 \%$ afirmaram que são filhas de mães solteiras. $51,5 \%$ das jovens tem uma renda familiar de até um salário mínimo e $91,1 \%$ conhecem outras jovens que engravidaram. Conclusão: As adolescentes têm iniciado uma vida sexual precocemente, sendo favorável à uma possível gravidez na adolescência. A baixa condição socioeconômica, o baixo grau de escolaridade da mãe e da adolescente e a um mal relacionamento familiar também são fatores que predispõem uma possível gestação na adolescência.

Palavras-chave: Gravidez na adolescência, Sexualidade, Serviços de planejamento familiar.

\section{ABSTRACT}

Objective: To analyze risk factors for pregnancy in adolescents aged 10 to 19 years in the Maternal and Child Specialized Reference Unit (UREMIA); verify the use of contraceptive methods in the population specified above; to verify the socioeconomic profile of the adolescents' family. Methods: Quantitative descriptive observational study, aimed at collecting data on the factors that lead adolescents to become pregnant. The population of this study is composed of pregnant adolescents, aged between 10 and 19 years old. Results: $30.4 \%$ had sex at the age of 13 and 14, 39.3\% answered that they are single. Most adolescents are aware of STIs $(72.7 \%)$ and $54.1 \%$ of these guidelines were given mainly at school. $54.6 \%$ said they used contraception. $48.8 \%$ have incomplete elementary education. $45.6 \%$ stated that they are the daughters of single mothers. $51.5 \%$ of young women have a family income of up to one minimum wage and $91.1 \%$ know other young women who became pregnant. Conclusion: Adolescents have started a sexual life early, favoring a possible pregnancy in adolescence. The low socioeconomic status, the low level of education of the mother and adolescent and a poor family relationship are also factoring that predispose a possible pregnancy in adolescence.

Key words: Pregnancy in adolescence, Sexuality, Family planning services.

\footnotetext{
${ }^{1}$ Centro Universitário Metropolitano da Amazônia (UNIFAMAZ), Belém - PA. *E-mail: ananda_maciel@hotmail.com

${ }^{2}$ Centro Universitário do Estado do Pará (CESUPA), Belém - PA.

3Universidade da Amazônia (UNAMA), Belém - PA.

${ }^{4}$ Universidade Estadual do Pará (UEPA), Belém - PA.
} 


\section{RESUMEN}

Objetivo: Analizar los factores de riesgo de embarazo en adolescentes de 10 a 19 años en la Unidad Especializada de Referencia Materno Infantil (UREMIA); verificar el uso de métodos anticonceptivos en la población especificada anteriormente; verificar el perfil socioeconómico de la familia de los adolescentes. Metodos: Estudio observacional descriptivo cuantitativo, destinado a recolectar datos sobre los factores que llevan a las adolescentes a quedarse embarazadas. La población de este estudio está compuesta por adolescentes embarazadas, con edades comprendidas entre 10 y 19 años. Resultados: $30.4 \%$ tuvo relaciones sexuales a la edad de 13 y 14 años, 39.3\% respondió que son solteros. La mayoría de los adolescentes conocen las ITS $(72,7 \%)$ y el $54,1 \%$ de estas pautas se administraron principalmente en la escuela. El 54,6\% dijo que usaban anticonceptivos. El 48.8\% tiene educación primaria incompleta. El 45,6\% declaró que son hijas de madres solteras. El $51.5 \%$ de las mujeres jóvenes tienen un ingreso familiar de hasta un salario mínimo y el $91.1 \%$ conoce a otras mujeres jóvenes que quedaron embarazadas. Conclusión: Los adolescentes han comenzado una vida sexual temprano, favoreciendo un posible embarazo en la adolescencia. El bajo nivel socioeconómico, el bajo nivel de educación de la madre y el adolescente y una mala relación familiar también son factores que predisponen a un posible embarazo en la adolescencia.

Palabras clave: Embarazo em adolescencia, Sexualidad, Servicios de panificación familiar.

\section{INTRODUÇÃO}

Todos os dias, cerca de 20 mil adolescentes com menos de 18 anos dão à luz em países subdesenvolvidos (FPNU, 2013). E em média 16 milhões de adolescentes parem todos os anos, sendo a maioria em países subdesenvolvidos e em desenvolvimento (WHO, 2018). Com isso, podemos afirmar que a gravidez na adolescência está presente em todo o mundo, atualmente esse problema está sendo estudado tanto no contexto nacional quanto no internacional (DINIZ E e KOLLER HS, 2012).

Quanto ao risco, a gravidez na juventude é um problema de Saúde Pública, devido as adolescentes terem maior risco de desenvolver pré-eclâmpsia, desproporção feto-pélvica, síndromes hipertensivas, partos prematuros, anemia, restrição do crescimento fetal, além de complicações decorrentes de abortos provocados e/ou pela falta assistência adequada. Nas jovens de 15 a 19 anos, a probabilidade de mortes ligadas à gravidez ou parto é duas vezes maior do que nas mulheres de 20 anos ou mais; entre as menores de 15 anos, esse risco é aumentado em 5 vezes (MOTA RS, 2012).

Adolescência, deriva da palavra em latim adolecere significa crescer. Essa é uma fase da vida humana, situada entre a infância e a vida adulta, durante esse período o indivíduo passa por inúmeras transformações corporais e comportamentais (OYAMADA LH, et al., 2014). Não se consegue definir com precisão o início e o fim da adolescência, pois ela varia de pessoa para pessoa, contudo de acordo com a Organização Mundial de Saúde (OMS), ela ocorre entre os 10 e 19 anos de idade (MENDES, 2011). No Brasil, de acordo com o estatuto da criança e do adolescente (ECA) Lei $n . \stackrel{0}{8.069 / 90}$, considera-se adolescente indivíduos entre doze e dezoito anos de idade.

Nacionalmente, podemos verificar que a maior incidência da gravidez na adolescência ocorre principalmente em adolescentes que possuem uma condição socioeconômica baixa, isso ocorre devido muitos fatores que podem estar associados, como o baixo grau de escolaridade e evasão escolar, estes costumam ser os fatores principais (MACHADO NO, et al., 2007).

Em 2011 uma pesquisa revelou o número de nascimentos por ocorrência e idade da mãe de acordo com as regiões brasileiras, o Norte ficou em segundo lugar em relação à ocorrência de gravidez na adolescência, de acordo com os dados levantados, de um total de 313.029 nascimentos, haviam 82.957 em que as parturientes tinham entre 10 a 19 anos (RNPI, 2014). No Pará, no ano de 2014 houve 143.503 nascimentos, sendo que em 39.138 nascimentos as mães tinham idade entre 10 a 19 anos (BRASIL, 2014).

Outro fator que predispõe à gravidez na adolescência são os problemas familiares, como a falta de comunicação entre os jovens e seus pais. Subtende-se que quando não se quer engravidar o percentual de prevenção é $100 \%$, contudo, existem as gravidezes indesejadas nota-se que essa prevenção não é efetiva (MOTA RS, 2012). É importante frisar que por vezes esses adolescentes não usam os métodos contraceptivos 
de forma adequada, devido ao entendimento limitado sobre prevenção à gravidez e a própria negação do adolescente em relação a possibilidade de engravidar (DIAS ACG, 2010).

Diante disso, a gravidez na adolescência é considerada um problema de saúde pública, visto que muitas vezes ela acontece devido a falta de panejamento familiar e a falta de educação sexual, que são assuntos ainda pouco discutidos em nossa sociedade (DINIZ E e KOLLER HS, 2012). A conversa entre os adolescentes, professores, profissionais da saúde e seus responsáveis pode ocasionar em uma melhora de compreensão em relação à vulnerabilidade relacionada à gravidez na adolescência (MELO J, et al., 2015).

Este estudo teve como objetivo analisar os fatores de risco que levam a incidência de gravidez em adolescentes na faixa etária de 10 a 19 anos no ano de 2017 na cidade de Belém, Pará, além de verificar a utilização de métodos contraceptivos na população acima especificada e o perfil socioeconômico da família das adolescentes.

\section{MÉTODOS}

Trata-se de um estudo quantitativo descritivo observacional realizado na cidade de Belém, no estado do Pará, que visa o levantamento de dados sobre os fatores que levam às adolescentes a engravidarem.

A população desse estudo foi composta por adolescentes grávidas, com idade entre 10 e 19 anos, atendidas pelo serviço público de saúde da cidade de Belém - PA, entre os meses de maio a setembro do ano de 2017. Para compor a amostra deste estudo piloto, conforme Fontelles MJ, et al. (2010), poderiam ser selecionadas, por conveniência, no mínimo 31 adolescentes grávidas. Contudo, todas as adolescentes grávidas em atendimento na Unidade de Referência no período em questão foram convidadas a participar da pesquisa e constituíram a amostra, de acordo com os critérios de inclusão estabelecidos.

Foram incluídos na pesquisa indivíduos adolescentes grávidas, com idade entre 10 e 19 anos, e que estivessem cadastradas na Unidade de Referência. Adolescentes menores de 18 anos só foram incluídas na pesquisa após receber a concordância da mesma por meio de assinatura do Termo de Assentimento Livre e Esclarecido (TALE), e dos pais ou do responsável legal por meio de assinatura do Termo de Consentimento Livre e Esclarecido (TCLE).

Para a coleta de dados foi utilizado um questionário semiestruturado autoaplicável contendo questões relacionadas aos aspectos sociodemográficos (idade, escolaridade, estado civil, renda familiar, número de moradores na residência), vida sexual (início da vida sexual, uso de métodos anticoncecionais, perceção sobre o uso de métodos anticoncecionais), aspectos familiares (número de irmãos, escolaridade e estado civil da mãe e relacionamento com os pais), conhecimento sobre as Infeções Sexualmente Transmissíveis (IST's) e aspectos relacionados à gravidez na adolescência (impacto da gravidez na vida escolar).

Cada questionário foi identificado por um número, preservando assim a identidade da adolescente. Os dados coletados foram organizados e tabulados em planilhas do programa Excel 2013 e, e a análise descritiva foi realizada por meio de cálculos de frequências absolutas e relativas.

Esta pesquisa respeitos os aspectos éticos e legais preconizados pela Resolução no 466/2012 do Conselho Nacional de Saúde (CNS) (BRASIL, 2012), que estabelece diretrizes para pesquisas envolvendo seres humanos, sendo aprovada pelo Comitê de Ética em Pesquisa (CEP) da Faculdade Metropolitana da Amazônia (FAMAZ), sob parecer ํo 2.339.987 e CAAE no 74569417.3.0000.5701.

\section{RESULTADOS}

Neste estudo piloto foram aplicados questionários a 33 gestantes. A idade variou de 14 a 18 anos ou mais, sendo a média de idade de 16,1 $\pm 1,2$ anos. Dentre elas, 30,4\% tiveram a sexarca aos 13 e 14 anos. Verificouse que quase metade das adolescentes possuíam ensino fundamental incompleto $(48,4 \%)$ ou ensino médio incompleto (30,3\%). Quanto ao estado civil, $54,5 \%$ responderam que são solteiras, $36,4 \%$ moram com o parceiro e apenas $9,09 \%$ são casadas. Em relação aos aspectos econômicos, observou-se que $51,5 \%$ das jovens têm renda familiar de até um salário mínimo. Também foi constatado que em 30,4\% delas moram com 5 pessoas na mesma casa (Tabela $\mathbf{1}$ ). 
Tabela 1 - Características sociodemográficas e econômicas das adolescentes participantes da pesquisa. Belém - PA, 2019.

\begin{tabular}{|c|c|c|}
\hline Variáveis & $\mathbf{N}$ & $\%$ \\
\hline \multicolumn{3}{|l|}{ Escolaridade } \\
\hline Ensino fundamental incompleto & 16 & 48,4 \\
\hline Ensino fundamental completo & 3 & 9,0 \\
\hline Ensino médio incompleto & 10 & 30,3 \\
\hline Ensino médio completo & 3 & 9,0 \\
\hline Ensino superior incompleto & 1 & 3,3 \\
\hline \multicolumn{3}{|l|}{ Estado civil } \\
\hline Solteira & 18 & 54,5 \\
\hline Casada & 3 & 9,1 \\
\hline Morando junto com parceiro & 12 & 36,4 \\
\hline \multicolumn{3}{|l|}{ Renda familiar } \\
\hline Até 1 salário mínimo & 17 & 51,5 \\
\hline De 1 a 2 salários mínimo & 13 & 39,4 \\
\hline De 2 a 4 salários mínimo & 3 & 9,1 \\
\hline \multicolumn{3}{|l|}{ Número de moradores na casa } \\
\hline 2 & 6 & 18,2 \\
\hline 3 & 5 & 15,1 \\
\hline 4 & 5 & 15,1 \\
\hline 5 & 10 & 30,4 \\
\hline 6 ou mais & 7 & 21,2 \\
\hline Total & 33 & $100 \%$ \\
\hline
\end{tabular}

Fonte: Paiva AM, et al., 2020.

Outro fator importante analisado foram os aspectos familiares, onde todas as participantes referiram ter irmãos, sendo que $27 \%$ alegou ter 5 ou mais irmãos. Em relação ao estado civil materno, $45,6 \%$ afirmaram que são filhas de mães solteiras. Além disso, 30,3\% das adolescentes disseram que suas mães possuem ensino fundamental incompleto, e $27,2 \%$ possuem ensino médio incompleto. Ao abordar o relacionamento familiar, $87,9 \%$ das gestantes relataram que possuem um bom relacionamento com seus pais, contudo, $57,6 \%$ afirmaram não se sentirem à vontade para falar sobre sexo com seus responsáveis.

Outros aspectos como o conhecimento de e o conhecimento acerca dos métodos contraceptivos foram abordados. Em relação às IST's, notou-se que a maioria das adolescentes tem conhecimento sobre o tema, totalizando $72,7 \%$ e que $54,1 \%$ destas orientações foram passadas principalmente em ambiente escolar.

Em relação aos métodos contraceptivos, 54,6\% afirmaram fazer uso de algum método, sendo mais relatado o uso de anticoncepcional injetável (73,4\%), em seguida o uso de camisinha (40\%). Ao classificar a qualidade do acesso aos métodos contraceptivos, 54,5\% avaliaram como bom e apenas 3,03\% classificaram como ruim. Em relação ao uso do preservativo, que é tanto um método contraceptivo quanto um método de prevenção de IST's, sua grande maioria $(75,8 \%)$ o classificou como um método seguro (Tabela 2 ). 
Tabela 2 - Percepções e prática das adolescentes participantes da pesquisa quanto ao uso de métodos contraceptivos. Belém - PA, 2019.

\begin{tabular}{lcc}
\hline Questões & N & \% \\
\hline Fazia uso de métodos contraceptivos antes de engravidar? & 18 & 54,6 \\
\hline Sim & 15 & 45,4 \\
Não & & \\
\hline Se sim, qual? & 11 & 73,4 \\
\hline Anticoncepcional injetável & 6 & 40 \\
Camisinha & 1 & 6,6 \\
Anticoncepcional via oral & & \\
\hline O que acha do acesso aos contraceptivos? & 5 & 15,1 \\
\hline Ótimo & 18 & 54,5 \\
Bom & 8 & 24,2 \\
Regular & 1 & 3,03 \\
Ruim & 1 & 3,03 \\
Sem resposta & & \\
\hline Acha camisinha um método seguro? & 25 & 75,8 \\
\hline Sim & 8 & 24,2 \\
Não & 33 & $\mathbf{1 0 0 , 0}$ \\
\hline Total & &
\end{tabular}

Fonte: Paiva AM, et al., 2020.

Vale ressaltar que $60,7 \%$ não interromperam os estudos por conta da gestação e das adolescentes que porventura precisaram interromper, é unanime (100\%) a intenção de retornar para a escola. As adolescentes também foram questionadas em relação a conhecerem outra jovem que engravidaram na adolescência e 90,1\% afirmaram que sim, em sua maioria, uma amiga próxima (Tabela $\mathbf{3}$ ).

Tabela 3 - Aspectos relacionados ao impacto da gravidez na vida das adolescentes participantes da pesquisa. Belém - PA, 2019.

\begin{tabular}{lcc}
\hline Questões & $\mathbf{N}$ & $\%$ \\
\hline Você interrompeu os estudos por conta da gravidez? & 13 & 39,3 \\
\hline Sim & 20 & 60,7 \\
Não & & \\
\hline Caso tenha, pretende retornar, ou já retornou a estudar? & 13 & 100,0 \\
\hline Sim & 0 & 0,0 \\
Não & & \\
\hline Conhece alguém que engravidou na adolescência? & 30 & 90,1 \\
\hline Sim & 3 & 9,9 \\
Não & $\mathbf{3 3}$ & $\mathbf{1 0 0 , 0}$ \\
\hline Total &
\end{tabular}

Fonte: Paiva AM, et al., 2020. 


\section{DISCUSSÃO}

A gravidez na adolescência é um fenômeno observado mundialmente que desafia os serviços de saúde pública devido ao seu difícil controle, afetando principalmente países em desenvolvimento, a exemplo do Brasil. Dessa forma, é essencial conhecer os fatores que propiciam esse evento na adolescência.

Neste estudo, observou-se que a maioria das adolescentes têm menos de 8 anos de estudo. Pinheiro YT, et al. (2019) investigaram os fatores associados à gravidez entre 234 adolescentes de João Pessoa, Paraíba e observaram $66,7 \%$ delas possuíam menos de 8 anos de estudo. Este resultado também foi encontrado no estudo de Hubert C, et al. (2019), os quais verificaram que $86,4 \%$ das adolescentes grávidas possuíam 8 ou menos anos de estudos. Por outro lado, Borovac-Pinheiro A, et al. (2019) observaram que mais de $45 \%$ das adolescentes grávidas de seu estudo possuíam ensino médio ou superior.

Segundo Hubec C, et al. (2019), a falta de acesso efetivo a níveis mais elevados de educação, o casamento precoce ou a entrada em uma união de coabitação e a vivência precoce na maternidade continuarão sendo, na maioria das vezes, como a única opção de vida para as adolescentes economicamente desfavorecidas.

Há de se assinalar que cerca de $36 \%$ das participantes afirmaram ser solteiras. Em estudo semelhante, Hubert C, et al. (2019) entrevistaram 1.639 mulheres jovens mexicanas entre 15 e 24 anos com o objetivo de avaliar os fatores associados à gravidez na adolescência e verificaram que mais de $70 \%$ das adolescentes grávidas que estavam na faixa etária de 15 a 19 anos eram solteiras. Este resultado, no entanto, contraria a maioria das pesquisas nacionais e internacionais, em que a maioria das adolescentes são casadas (SANTOS LAV, et al., 2018; PINHEIRO Y, et al., 2019; BOROVAC-PINHEIRO A, et al., 2019; HUBERT C, et al., 2019).

No que concerne a questões socioeconômicas, a média salarial das gestantes está em uma faixa de 1,5 salários mínimos. Ademais, deve-se levar em consideração que o grupo pesquisado possui dois entraves no tangente à empregabilidade: a baixa idade e a condição restritiva a exercer atividade remunerada. Neste sentido, surge a preocupação relacionada à estrutura familiar e à criação do recém-nascido, visto ser imprescindível o apoio familiar para o bom desenvolvimento da gestação, uma vez que a futura mãe sente medo da reação dos pais diante da sua gravidez indesejada, além de existir o aumento das dificuldades socioeconômicas para criação do novo membro da família (MOREIRA TM, et al., 2008).

Dessa forma, cerca de $88 \%$ das entrevistadas afirmaram possuir boa relação com seus pais, constituindo um número de grande relevância para amenizar tais entraves presentes na gestação precoce. Uma pesquisa que analisou a vivência da gravidez na adolescência no âmbito familiar e social na cidade de Santa Maria, Rio Grande do Sul, evidenciou que quase a totalidade das adolescentes grávidas tinham um bom relacionamento com a família, especialmente com a mãe, que geralmente é a primeira a receber a notícia da gravidez (SANTOS CC, et al., 2014). De acordo com Vieira APR, et al. (2013), o apoio familiar é fundamental para desmistificar medos e angústias relacionados à gravidez, além de contribuir para a superação das dificuldades no cuidado com a criança, favorecendo o desenvolvimento da maternidade.

Santos CC, et al. (2014), argumentam que as mães das adolescentes são os principais elementos na rede de apoio, sendo consideradas pelas jovens como a maior fonte de apoio social tanto no nível emocional como no instrumental e afetivo, por serem mais compreensíveis à problemática vivenciada pelas filhas. $O$ apoio materno possibilita a adolescente a construção de capacidades para lidar com as novas relações que se estabelecem em decorrência da gestação

Ademais, sobre o apoio familiar, há de se mencionar que uma boa relação entre o binômio pais-filho é fundamental para a transmissão de conhecimento relacionados a temáticas delicadas como a sexualidade, que é, por vezes, tida como tabu entre a sociedade contemporânea.

Em relação a isto, há de se levar em consideração que cerca de $57 \%$ das entrevistadas afirmaram não se sentirem confortáveis em falar sobre sexualidade com seus pais. Em estudo realizado por Pereira FAF, et al. (2017) foi destacado que a presença de uma relação próxima entre mãe e filha é fundamental para a prevenção de gravidez precoce, pois tal relação possibilita o diálogo antes do período de início de atividade sexual da filha. 
Destarte, após o nascimento as adolescentes e famílias não dispõem de recursos humanos e financeiros para deixar o recém-nascido sob cuidados de uma cuidadora passando a dedicar todo seu tempo as tarefas e cuidados exigidos pelo recém-nascido. $O$ abandono e a falta de escolaridade podem refletir no futuro se transformar em dependência econômica que a adolescente vai ter do companheiro ou da família e, se ela pretender se inserir no mercado de trabalho, fará em desvantagem em relação aos que alcançaram certo grau de estudo, contribuindo, dessa forma, para a perpetuação da desigualdade existente (GOMES AM, et al., 2015).

Todavia, ao falar de apoio familiar, deve-se transcender ao pensamento restrito às questões socioeconômicas, visto tratar-se de uma população que é submetida duplamente a alterações severas, isto é, às alterações típicas da adolescência, e às alterações biopsicossociais da gestação e puerpério.

A transição abrupta da adolescência e o encurtamento das experiências advindas desta fase, aliadas a um processo gestacional pode causar, transtornos mentais significativos (NOGUEIRA SM e MENDONÇA JB, 2015). Além disto, o desamparo familiar é fator de risco para a potencialização destes transtornos, conforme elucidado por Rodrigues SMS, et al. (2017), sendo comum entre as grávidas desamparadas pela sua família a prevalência de transtornos mentais comuns, especialmente em casos de gravidez indesejada, como no caso das ocorridas durante a adolescência. Portanto, gravidez na adolescência não deve ser vista somente de forma negativa, pois esta pode ser até mesmo uma forma de amadurecimento, mesmo que precoce para grande parte das adolescentes e familiares, desde que a adolescente cumpra suas responsabilidades maternas (SILVA L e TONNETE VLP, 2006).

Santos TMB, et al. (2015), em uma revisão sistemática de literatura, implicam que os homens nessa faixa etária afirmam ser dotados de superior inteligência a das garotas e que, a eles, o sexo é permitido independentemente de algum vínculo afetivo, tendo maior liberdade sexual e que devido a isso delegam a elas a responsabilidade do uso de contraceptivos. Porém, os próprios garotos pressupõem que as meninas não podem adotar condutas tão liberais, e que para elas o sexo deve ser mais restrito. Logo, percebe-se o papel fundamental da educação sexual nas escolas e unidades de saúde, que será abordado adiante.

No que se refere à sexarca, afirma-se que há uma prevalência significativa de sexualização precoce, onde observou-se que cerca de um terço das adolescentes $(30,4 \%)$ tiveram um início precoce da vida sexual, entre os 13 e 14 anos. Um estudo envolvendo 129 adolescentes da cidade de Campinas, São Paulo, também descobriu que a maioria das adolescentes tinham iniciado a vida sexual entre 13 e 14 anos, no entanto, com prevalência superior à do presente estudo (54,5\%) (BOROVAC-PINHEIRO A, et al., 2019).

Tais dados mostram-se preocupantes, visto a iniciação sexual precoce estar relacionado à transmissão de IST's, uso incorreto de métodos contraceptivos e/ou gravidez precoce (LARA LAS, 2015). Além disso, a sociedade também impulsiona o ato sexual no adolescente, estimulando no mesmo a vida sexual precoce (DOMINGOS AC, 2010).

Santos TMB, et al. (2015) relatam que os adolescentes são induzidos a iniciar a vida sexual precocemente por motivos diferentes, a sexarca no homem tem papel de autoafirmação perante a sociedade e na mulher é diferente, Ihe é induzida a postergar a primeira relação e quando o fizer deve estar em uma vida sexual monogâmica, com parceiro estável.

O conhecimento sobre as IST's é de grande importância no quadro de gravidez na adolescência. Considerando o número de entrevistadas, $72,7 \%$ das mesmas afirmaram conhecer sobre esta temática, constituindo um número de grande relevância. Segundo Gonçalves RC, et al. (2013), as IST's representam uma séria problemática acerca da saúde reprodutiva dos jovens, pois interferem de diversas formas nos mesmos, podendo causar esterilidade além de influenciar negativamente a sua autoestima. Diante disso, o conhecimento empregado pela família $(25 \%)$, escola $(54,1 \%)$, USF $(20,8 \%)$ e UREMIA $(4,1 \%)$ é de suma importância para o adolescente exercer sua sexualidade de forma segura.

Corrêa S, et al. (2015) cita que, em 1988, a Organização Mundial da Saúde adotou o conceito de "saúde reprodutiva". A assistência à saúde reprodutiva é definida como conjunto de métodos, serviços e técnicas que contribuem para a saúde e o bem-estar reprodutivo, resolvendo e prevenindo os problemas de saúde 
reprodutiva. Isto inclui igualmente a saúde sexual, que tem com finalidade a melhoria da qualidade de vida e das relações pessoais e não somente aconselhamento e assistência relativos à reprodução e às infecções sexualmente transmissíveis. Nesse aspecto, a educação sexual nas escolas e unidades de saúde tem se mostrado eficaz ao trazer esses assuntos ao conhecimento da população.

Outro aspecto relacionado ao quadro de gravidez precoce é o uso de métodos contraceptivos. Mais da metade $(54,6 \%)$ das adolescentes grávidas fez o uso de algum dos métodos existentes, sendo o anticoncepcional injetável o mais utilizado (73,4\%). O estudo de Pinheiro PNC, et al. (2011) mostrou que os adolescentes conhecem estes métodos, entretanto, isto não significa que eles sabem usá-los, sendo necessário tal conhecimento para evitar IST's e a gravidez precoce indesejada.

Dessa forma, devem participar da educação sexual dos jovens: educadores de tal forma que a escola não tratará a sexualidade como um tabu (MOIZÉS JS e BUENO SMV, 2010), a família a qual atuará como a base para o adolescente, e o profissional da saúde o qual atuará na promoção da saúde (BORGES ALV, et al., 2006).

Um fator diretamente associado à temática "gravidez na adolescência" diz respeito à escolaridade da mãe durante o período gestacional. Neste sentido, aproximadamente $48 \%$ das entrevistadas possuíam o ensino fundamental incompleto, enquanto apenas 3\% possuía o Ensino Superior incompleto. Tal cenário dispare evidencia a dificuldade comum em conciliar os cuidados gestacionais à rotina estudantil e às condições socioeconômicas da família. Dentro das entrevistadas cerca de $40 \%$ afirmaram ter abandonado os estudos por conta da gestação, todavia, é unânime entre estas o interesse em retornar aos estudos.

Gomes AM, et al. (2015) relata que, é indubitável que os transtornos físicos advindos da gravidez exigem um tratamento especial por parte das instituições de ensino, entretanto sabe-se que quando eles são respeitados e tratados de maneira adequada, são habilmente superados pela puérpera sem que venham a interferir drasticamente nas suas atividades diárias.

As escolas têm que se preparar para essa demanda, tanto em relação ao aspecto físico quanto psicológico. Sob outra perspectiva, é essencial que os educadores e as alunas sejam devidamente informados dos direitos assegurados por lei a elas, para que a gravidez precoce não seja mais relacionada ao abandono escolar. Além disso, um maior conhecimento quanto aos métodos contraceptivos está diretamente relacionado a escolaridade, o que pode ainda evitar uma reincidência de gravidez na adolescência.

Uma das principais limitações do estudo foi a aceitação das adolescentes em participar da pesquisa, por vergonha ou por tempo indisponível pelas mesmas para conciliar uma nova rotina, dividida por vezes entre a realização de consultas de pré-natal, estudo e atividades diárias. Portanto, seria ideal em novas pesquisas sobre o tema, uma abordagem em um setor menos formal, onde um vínculo possa ser firmado mais facilmente.

Isso que poderia ser feito através de visitas domiciliares ou mesmo através da parceria com as escolas, conferindo assim um suporte mais amplo e com apoio psicológico necessário a estas adolescentes. Portanto, novos estudos, com amostras mais significativas devem ser realizados para melhor identificar as condições associadas à gravidez na adolescência, possibilitando assim, a identificação de estratégias para a sua redução.

\section{CONCLUSÃO}

Os resultados encontrados neste estudo sugerem que as adolescentes têm iniciado uma vida sexual precocemente, fator este favorável à uma possível gravidez ainda na adolescência. A baixa condição socioeconômica da adolescente, associada ao baixo grau de escolaridade da mãe e a falta de diálogo com a família a respeito de assuntos relacionados ao sexo e sexualidade também são fatores que predispõem uma possível gestação na adolescência, visto que essas condições estão relacionadas com uma possível desestruturação familiar, acarretando uma orientação precária destas jovens. Diante disso, considera-se que existe uma necessidade de incentivo para que exista uma melhor orientação sexual para as adolescentes, principalmente em âmbito familiar. 


\section{REFERÊNCIAS}

1. ALVES ED, et al. Estudos sobre gravidez na adolescência: a constatação de um problema social. Journal of Health Sciences, 2015; 12(3): 49-56.

2. BOROVAC-PINHEIRO A, et al. Empowering adolescent mothers in the choice of contraceptive methods at the postpartum period: avoiding a subsequent pregnancy. Rev. Bras. Ginecol. Obstet., 2019; 41(10):607-612.

3. BRASIL. Conselho Nacional de Saúde. Resolução no 466, de 12 de dezembro do 2012. Brasília: Diário Oficial da União; 2012.

4. DIAS ACG, TEIXEIRA MAP. Gravidez na adolescência: um olhar sobre um fenômeno complexo. Paidéia (Ribeirão Preto), 2010; 20(45):123-31.

5. DINIZ E, KOLLER HS. Fatores associados à gravidez em adolescentes brasileiros de baixa renda. Paidéia (Ribeirão Preto), 2012; 22(53):305-14.

6. FONTELLES MJ, et al. Metodologia da pesquisa: diretrizes para o cálculo do tamanho da amostra. Rev Paran Med., 2010;24:57-64.

7. GOMES, AM. O impacto da gravidez na escolaridade das adolescentes. InterMeio: Revista do Programa de PósGraduação em Educação-UFMS, 2016; 4(7):46-59.

8. HENRIQUE L, GODINHO TM. Gravidez na adolescência e o risco para a gestante. Brazilian Journal of Surgery and Clinical Research, 2014; 6(2):38-45.

9. HUBERT C, et al. Factors associated with pregnancy and motherhood among Mexican women aged 15-24. Cad. Saúde Pública, 2019; 35(6):e00142318.

10. LARA LAS, et al. Aspectos da atividade sexual precoce. Revista Brasileira de Ginecologia e Obstetrícia, 2015; 37(5):199-202.

11. LONGO LAFB. Juventude e contracepção: um estudo dos fatores que influenciam o comportamento contraceptivo das jovens brasileiras de 15 a 24 anos. Revista Brasileira de Estudos de População, 2002; 19(2):229-47.

12. MACHADO NO, et al. Características socio-demográficas e reprodutivas de adolescentes atendidas no pós-parto no Instituto da Criança da Universidade de São Paulo. Revista Brasileira de Crescimento e Desenvolvimento Humano, 2007;17(3):1-7.

13. MELO J, et al. Gravidez na adolescência: realidade e repercussões sobre atividade sexual. INTENSA, 2015; 9(2):1622.

14. MENDES SS, et al. Saberes e atitudes dos adolescentes frente à contracepção. Revista Paulista de Pediatria, 2011; 29(3):385-91.

15. MOTA RS. História oral de adolescentes grávidas em situação de violência doméstica. Dissertação (Mestrado em Enfermagem) - Escola de Enfermagem. Universidade Federal da Bahia, Salvador, 2012; 94p.

16. NOGUEIRA SM, MENDONÇA JB. Fatores de risco para desenvolvimento de transtornos mentais comuns em adolescentes gestantes do município de Ceres-GO. Revista Eletrônica da Faculdade de Ceres, 2015; 4(2):54-65.

17. OYAMADA LH, et al. Gravidez na adolescência e o risco para a gestante. BJSCR - Brazilian Journal of Surgery and Clinical Research, v. 6, n. 2, p. 38-45, mar/mai, 2014.

18. PEREIRA FAF, et al. Desafio das mulheres que foram mães na adolescência quanto a prevenção da gravidez precoce de suas filhas. Unimontes Científica, 2017; 19(2):73-86.

19. PINHEIRO YT, et al. Fatores associados à gravidez em adolescentes de um município do nordeste do Brasil. Cad. saúde colet., 2019;27(4):363-367.

20. RODRIGUES SMS, et al. Suporte familiar e transtornos mentais comuns em adolescentes grávidas. Psicologia Argumento, 2017; 29(64):91-100.

21. SANTOS CC, et al. A vivência da gravidez na adolescência no âmbito familiar e social. Rev Enferm UFSM, 2014;4(1):105-112.

22. SANTOS LAV, et al. História gestacional e características da assistência pré-natal de puérperas adolescentes e adultas em uma maternidade do interior de Minas Gerais, Brasil. Ciênc. saúde coletiva, 2018;23(2):617-625.

23. SANTOS TMB, et al. Fatores que contribuem para o início da atividade sexual em adolescentes: revisão integrativa. Revista de Atenção à Saúde, 2015; 13(44):64-70.

24. VIEIRA APR, et al. Maternidade na adolescência e apoio familiar: implicações no cuidado materno à criança e autocuidado no puerpério. Cienc Cuid Saude, 2013;12(4):679-68. 